\title{
Fixed point theorems on ordered gauge spaces with applications to nonlinear integral equations
}

Meryam Cherichi ${ }^{1}$ and Bessem Samet ${ }^{2^{*}}$

\author{
* Correspondence: bessem. \\ samet@gmail.com \\ ${ }^{2}$ Ecole Supérieure des Sciences et \\ Techniques de Tunis, 5, Avenue \\ Taha Hussein-Tunis, B.P. 56, Bab \\ Menara 1008, Tunisie \\ Full list of author information is \\ available at the end of the article
}

\begin{abstract}
We establish coincidence and fixed point theorems for mappings satisfying generalized weakly contractive conditions on the setting of ordered gauge spaces. Presented theorems extend and generalize many existing studies in the literature. We apply our obtained results to the study of existence and uniqueness of solutions to some classes of nonlinear integral equations.
\end{abstract}

Keywords: Gauge spaceordered setcoincidence pointfixed pointaltering distance function

\section{Introduction}

Fixed point theory is considered as one of the most important tools of nonlinear analysis that widely applied to optimization, computational algorithms, physics, variational inequalities, ordinary differential equations, integral equations, matrix equations and so on (see, for example, [1-6]). The Banach contraction principle [7] is a fundamental result in fixed point theory. It consists of the following theorem.

Theorem 1.1 (Banach [7]) Let $(X, d)$ be a complete metric space and let $T: X \rightarrow X$ be a contraction, i.e., there exists $k \in[0,1)$ such that $d(T x, T y) \leq k d(x, y)$ for all $x, y \in$ $X$. Then $T$ has a unique fixed point, that is, there exists a unique $x^{*} \in X$ such that $T x^{*}=x^{*}$. Moreover, for any $x \in X$, the sequence $\left\{T^{n} x\right\}$ converges to $x^{*}$.

Generalization of the above principle has been a heavily investigated branch of research (see, for example, [8-10]). In particular, there has been a number of studies involving altering distance functions. There are control functions which alter the distance between two points in a metric space. Such functions were introduced by Khan et al. [11], where they present some fixed point theorems with the help of such functions.

Definition 1.1 An altering distance function is a function $\psi:[0, \infty) \rightarrow[0, \infty)$ which satisfies

(a) $\psi$ is continuous and nondecreasing;

(b) $\psi(t)=0$ if and only if $t=0$.

In [11], Khan et al. proved the following result.

Theorem 1.2 (Khan et al. [11]) Let $(X, d)$ be a complete metric space, $\psi$ be an altering distance function, $c \in[0,1)$ and $T: X \rightarrow X$ satisfying

$$
\psi(d(T x, T y)) \leq c \psi(d(x, y))
$$

(C) 2012 Cherichi and Samet; licensee Springer. This is an Open Access article distributed under the terms of the Creative Commons Attribution License (http://creativecommons.org/licenses/by/2.0), which permits unrestricted use, distribution, and reproduction in any medium, provided the original work is properly cited. 
for all $x, y \in X$. Then $T$ has an unique fixed point.

Altering distance has been used in metric fixed point theory in many studies (see, for example, [2,3,12-19]). On the other hand, Alber and Guerre-Delabriere in [12] introduced a new class of contractive mappings on closed convex sets of Hilbert spaces, called weakly contractive maps.

Definition 1.2 (Alber and Guerre-Delabriere [12]) Let $(E,\|\cdot\|)$ be a Banach space and $C \subseteq E$ a closed convex set. A map $T: C \rightarrow C$ is called weakly contractive if there exists an altering distance function $\psi:[0, \infty) \rightarrow[0, \infty)$ with $\lim _{t \rightarrow \infty} \psi(t)=\infty$ such that

$$
\|T x-T y\| \leq\|x-y\|-\psi(\|x-y\|),
$$

for all $x, y \in X$.

In [12], Alber and Guerre-Delabriere proved the following result.

Theorem 1.3 (Alber and Guerre-Delabriere [12]) Let $H$ be a Hilbert space and $C \subseteq$ $H$ a closed convex set. If $T: C \rightarrow C$ is a weakly contractive map, then it has a unique fixed point $x^{*} \in C$.

Rhoades [18] proved that the previous result is also valid in complete metric spaces without the condition $\lim _{t \rightarrow \infty} \psi(t)=\infty$.

Theorem 1.4 (Rhoades [18]) Let $(X, d)$ be a complete metric space, $\psi$ be an altering distance function and $T: X \rightarrow X$ satisfying

$$
d(T x, T y) \leq d(x, y)-\psi(d(x, y))
$$

for all $x, y \in X$. Then $T$ has a unique fixed point.

Dutta and Choudhury [20] present a generalization of Theorems 1.2 and 1.4 proving the following result.

Theorem 1.5 (Dutta and Choudhury [20]) Let $(X, d)$ be a complete metric space and $T: X \rightarrow X$ be a mapping satisfying

$$
\psi(d(T x, T y)) \leq \psi(d(x, y))-\varphi(d(x, y))
$$

for all $x, y \in X$, where $\psi$ and $\phi$ are altering distance functions. Then $T$ has an unique fixed point.

An extension of Theorem 1.5 was considered by Dorić [13].

Theorem 1.6 (Dorić [13]) Let $(X, d)$ be a complete metric space and $T: X \rightarrow X$ be a mapping satisfying

$$
\psi(d(T x, T y)) \leq \psi(M(x, y))-\varphi(M(x, y)),
$$

for all $x, y \in X$, where

$$
M(x, y)=\max \left\{d(x, y), d(T x, x), d(T y, y), \frac{1}{2}[d(y, T x)+d(x, T y)]\right\},
$$

$\psi$ is an altering distance function and $\phi$ is a lower semi-continuous function with $\phi(t)=0$ if and only if $t=0$. Then $T$ has a unique fixed point.

Very recently, Eslamian and Abkar [14] (see also, Choudhury and Kundu [2]) introduced the concept of $(\psi, \alpha, \beta)$-weak contraction and established the following result.

Theorem 1.7 (Eslamian and Abkar [14]) Let $(X, d)$ be a complete metric space and $T: X \rightarrow X$ be a mapping satisfying 


$$
\psi(d(T x, T y)) \leq \alpha(d(x, y))-\beta(d(x, y))
$$

for all $x, y \in X$, where $\psi, \alpha, \beta:[0, \infty) \rightarrow[0, \infty)$ are such that $\psi$ is an altering distance function, $\alpha$ is continuous, $\beta$ is lower semi-continuous,

$$
\alpha(0)=\beta(0)=0 \text { and } \psi(t)-\alpha(t)+\beta(t)>0 \text { for all } t>0 .
$$

Then $T$ has a unique fixed point.

Note that Theorem 1.7 seems to be new and original. Unfortunately, it is not the case. Indeed, the contractive condition (1) can be written as follows:

$$
\psi(d(T x, T y)) \leq \psi(d(x, y))-\varphi(d(x, y)),
$$

where $\phi:[0, \infty) \rightarrow[0, \infty)$ is given by

$$
\varphi(t)=\psi(t)-\alpha(t)+\beta(t), \quad t \geq 0 .
$$

Clearly, from the hypotheses of Theorem 1.7, the function $\phi$ is lower semi-continuous with $\phi(t)=0$ if and only if $t=0$. So Theorem 1.7 is similar to Theorem 1.6 of Dorić [13].

On the other hand, Ran and Reurings [6] proved the following Banach-Caccioppoli type principle in ordered metric spaces.

Theorem 1.8 (Ran and Reurings [6]) Let $(X, \preccurlyeq)$ be a partially ordered set such that every pair $x, y \in X$ has a lower and an upper bound. Let $d$ be a metric on $X$ such that the metric space $(X, d)$ is complete. Let $f: X \rightarrow X$ be a continuous and monotone (i.e., either decreasing or increasing with respect to $\preccurlyeq)$ operator. Suppose that the following two assertions hold:

1. there exists $k \in[0,1)$ such that $d(f x, f y) \leq k d(x, y)$ for each $x, y \in X$ with $x \leqslant y$;

2. there exists $x_{0} \in X$ such that $x_{0} \leqslant f x_{0}$ or $x_{0} \geqslant f x_{0}$.

Then $f$ has an unique fixed point $x^{*} \in X$.

Nieto and Rodríguez-López [4] extended the result of Ran and Reurings for non-continuous mappings.

Theorem 1.9 (Nieto and Rodŕiguez-López [4]) Let $(X, \preccurlyeq)$ be a partially ordered set and suppose that there exists a metric $d$ in $X$ such that the metric space $(X, d)$ is complete. Let $T: X \rightarrow X$ be a nondecreasing mapping. Suppose that the following assertions hold:

1. there exists $k \in[0,1)$ such that $d(T x, T y) \leq k d(x, y)$ for all $x, y \in X$ with $x \leqslant y$;

2. there exists $x_{0} \in X$ such that $x_{0} \leqslant T x_{0}$;

3. if $\left\{x_{n}\right\}$ is a nondecreasing sequence in $X$ such that $x_{n} \rightarrow x \in X$ as $n \rightarrow \infty$, then $x_{n} \leqslant x$ for all $n$.

Then $T$ has a fixed point.

Since then, several authors considered the problem of existence (and uniqueness) of a fixed point for contraction type operators on partially ordered metric spaces (see, for example, $[2,3,5,15-17,19,21-38])$.

In [3], Harjani and Sadarangani extended Theorem 1.5 of Dutta and Choudhury [20] to the setting of ordered metric spaces.

Theorem 1.10 (Harjani and Sadarangani [3]) Let $(X, \preccurlyeq)$ be a partially ordered set and suppose that there exists a metric $d$ in $X$ such that $(X, d)$ is a complete metric space. Let $T: X \rightarrow X$ be a nondecreasing mapping such that 


$$
\psi(d(T x, T y)) \leq \psi(d(x, y))-\varphi(d(x, y))
$$

for all $x, y \in X$ with $x \leqslant y$, where $\psi$ and $\phi$ are altering distance functions. Also suppose either

(I) $T$ is continuous or

(II) If $\left\{x_{n}\right\} \subset X$ is a nondecreasing sequence with $x_{n} \rightarrow x \in X$, then $x_{n} \leqslant x$ for all $n$.

If there exists $x_{0} \in X$ with $x_{0} \leqslant T x_{0}$, then $T$ has a fixed point.

In [16], Jachymski established a nice geometric lemma and proved that Theorem 1.10 of Harjani and Sadarangani can be deuced from an earlier result of O'Regan and Petruşel [33].

In this article, we present new coincidence and fixed point theorems in the setting of ordered gauge spaces for mappings satisfying generalized weak contractions involving two families of functions. Presented theorems extend and generalize many existing results in the literature, in particular Harjani and Sadarangani [3, Theorem 1.10], Nieto and Rodríguez-López [4, Theorem 1.9], Ran and Reurings [6, Theorem 1.8], and Dorić [13, Theorem 1.6]. As an application, existence results for some integral equations on the positive real axis are given.

Now, we shall recall some preliminaries on ordered gauge spaces and introduce some definitions.

\section{Preliminaries}

Definition 2.1 Let $X$ be a nonempty set. A map $d: X \times X \rightarrow[0, \infty)$ is called a pseudometric in $X$ whenever

(i) $d(x, x)=0$ for all $x \in X$;

(ii) $d(x, y)=d(y, x)$ for all $x, y \in X$;

(iii) $d(x, y) \leq d(x, z)+d(z, y)$ for all $x, y, z \in X$.

Definition 2.2 Let $X$ be a nonempty set endowed with a pseudo-metric d. The d-ball of radius $\varepsilon>0$ centered at $x \in X$ is the set

$$
B(x ; d, \varepsilon)=\{y \in X \mid d(x, y)<\varepsilon\} .
$$

Definition 2.3 A family $\mathcal{F}=\left\{d_{\lambda} \mid \lambda \in \mathcal{A}\right\}$ of pseudo-metrics is called separating if for each pair $x \neq y$, there is a $d_{\lambda} \in \mathcal{F}$ such that $d_{\lambda}(x, y) \neq 0$.

Definition 2.4 Let $X$ be a nonempty set and $\mathcal{F}=\left\{d_{\lambda} \mid \lambda \in \mathcal{A}\right\}$ be a separating family of pseudo-metrics on $X$. The topology $\mathcal{T}(\mathcal{F})$ having for a subbasis the family

$$
\mathcal{B}(\mathcal{F})=\left\{B\left(x ; d_{\lambda}, \varepsilon\right) \mid x \in X, d_{\lambda} \in \mathcal{F}, \varepsilon>0\right\}
$$

of balls is called the topology in $X$ induced by the family $\mathcal{F}$. The pair $(X, \mathcal{T}(\mathcal{F}))$ is called a gauge space. Note that $(X, \mathcal{T}(\mathcal{F}))$ is Hausdorff because we require $\mathcal{F}$ to be separating.

Definition 2.5 Let $(X, \mathcal{T}(\mathcal{F})$ ) be a gauge space with respect to the family $\mathcal{F}=\left\{d_{\lambda} \mid \lambda \in \mathcal{A}\right\}$ of pseudo-metrics on $X$. Let $\left\{x_{n}\right\}$ be a sequence in $X$ and $x \in X$.

(a) The sequence $\left\{x_{n}\right\}$ converges to $x$ if and only if

$$
\forall \lambda \in \mathcal{A}, \quad \forall \varepsilon>0, \quad \exists N \in N \mid d_{\lambda}\left(x_{n}, x\right)<\varepsilon, \quad \forall n \geq N .
$$

In this case, we denote $x_{n} \stackrel{\mathcal{F}}{\rightarrow} x$. 
(b) The sequence $\left\{x_{n}\right\}$ is Cauchy if and only if

$$
\forall \lambda \in \mathcal{A}, \quad \forall \varepsilon>0, \quad \exists N \in \mathbb{N} \mid d_{\lambda}\left(x_{n+p}, x_{n}\right)<\varepsilon, \quad \forall n \geq N, p \in \mathbb{N} .
$$

(c) $(X, \mathcal{T}(\mathcal{F}))$ is complete if and only if any Cauchy sequence in $(X, \mathcal{T}(\mathcal{F}))$ is convergent to an element of $X$.

(d) A subset of $X$ is said to be closed if it contains the limit of any convergent sequence of its elements.

Definition 2.6 Let $\mathcal{F}=\left\{d_{\lambda} \mid \lambda \in \mathcal{A}\right\}$ be a family of pseudo-metrics on $X .(X, \mathcal{F}, \preccurlyeq)$ is called an ordered gauge space if $(X, \mathcal{T}(\mathcal{F}))$ is a gauge space and $(X, \preccurlyeq)$ is a partially ordered set.

For more details on gauge spaces, we refer the reader to [39].

Now, we introduce the concept of compatibility of a pair of self mappings on a gauge space.

Definition 2.7 Let $(X, \mathcal{T}(\mathcal{F})$ ) be a gauge space and $f, g: X \rightarrow X$ are giving mappings. We say that the pair $\{f, g\}$ is compatible if for all $\lambda \in \mathcal{A}, d_{\lambda}\left(f g x_{n}, g f x_{n}\right) \rightarrow 0$ as $n$ $\rightarrow \infty$ whenever $\left\{x_{n}\right\}$ is a sequence in $X$ such that $f x_{n} \stackrel{\mathcal{F}}{\rightarrow} t$ and $g x_{n} \stackrel{\mathcal{F}}{\rightarrow} t$ for some $t \in X$.

Definition 2.8 (Ćirić et al. [29]) Let $(X, \preccurlyeq)$ be a partially ordered set and $f, g: X \rightarrow$ $X$ are two giving mappings. The mapping $f$ is said to be g-nondecreasing if for all $x, y \in$ $X$, we have

$$
g x \preccurlyeq g y \Rightarrow f x \preccurlyeq f y .
$$

Definition 2.9 Let $(X, \preccurlyeq)$ be a partially ordered set. We say that $(X, \preccurlyeq)$ is directed if every pair of elements has an upper bound, that is, for every $a, b \in X$, there exists $c \in$ $\mathrm{X}$ such that $a \leqslant c$ and $b \leqslant c$.

\section{Main results}

Let $(X, \mathcal{T}(\mathcal{F}))$ be a gauge space.

We consider the class of functions $\left\{\psi_{\lambda}\right\}_{\lambda \in \mathcal{A}}$ and $\left\{\varphi_{\lambda}\right\}_{\lambda \in \mathcal{A}}$ such that for all $\lambda \in \mathcal{A}$, $\psi_{\lambda}, \phi_{\lambda}:[0, \infty) \rightarrow[0, \infty)$ satisfy the following conditions:

(C1) $\psi_{\lambda}$ is an altering distance function.

(C2) $\phi_{\lambda}$ is a lower semi-continuous function with $\phi_{\lambda}(t)=0$ if and only if $t=0$.

Our first result is the following.

Theorem 3.1 Let $(X, \mathcal{F}, \preccurlyeq)$ be an ordered complete gauge space and let $f, g: X \rightarrow X$ be two continuous mappings such that $f$ is $g$-nondecreasing, $f(X) \subseteq g(X)$ and the pair $\{$, $g\}$ is compatible. Suppose that

$$
\psi_{\lambda}\left(d_{\lambda}(f x, f y)\right) \leq \psi_{\lambda}\left(d_{\lambda}(g x, g y)\right)-\varphi_{\lambda}\left(d_{\lambda}(g x, g y)\right)
$$

for all $\lambda \in \mathcal{A}$, for all $x, y \in X$ for which $g x \leqslant$ gy. If there exists $x_{0}$ such that $g x_{0} \leqslant f x_{0}$, then $f$ and $g$ have a coincidence point, that is, there exists a $z \in X$ such that $f z=g z$.

Proof. Let $x_{0} \in X$ such that $g x_{0} \leqslant f x_{0}$ (such a point exists by hypothesis). Since $f(X)$ $\subseteq g(X)$, we can choose $x_{1} \in X$ such that $f x_{0}=g x_{1}$. Then $g x_{0} \preccurlyeq f x_{0}=g x_{1}$. As $f$ is $g$-nondecreasing, we get $f x_{0} \leqslant f x_{1}$. Continuing this process, we can construct a sequence $\left\{x_{n}\right\}$ in $X$ such that

$$
g x_{n+1}=f x_{n}, \quad n=0,1, \ldots
$$


for which

$$
g x_{0} \preccurlyeq f x_{0}=g x_{1} \preccurlyeq f x_{1}=g x_{2} \preccurlyeq \cdots \preccurlyeq f x_{n-1}=g x_{n} \preccurlyeq \cdots
$$

Then from (2), for all $p, q \in \mathbb{N}$, for all $\lambda \in \mathcal{A}$, we have

$$
\psi_{\lambda}\left(d_{\lambda}\left(f x_{p}, f x_{q}\right)\right) \leq \psi_{\lambda}\left(d_{\lambda}\left(g x_{p}, g x_{q}\right)\right)-\varphi_{\lambda}\left(d_{\lambda}\left(g x_{p}, g x_{q}\right)\right) .
$$

We complete the proof in the following three steps.

Step 1. We will prove that

$$
d_{\lambda}\left(f x_{n}, f x_{n+1}\right) \rightarrow 0 \quad \text { as } n \rightarrow+\infty, \text { for all } \lambda \in \mathcal{A} .
$$

Let $\lambda \in \mathcal{A}$. We distinguish two cases.

- First case: We suppose that there exists $m \in \mathbb{N}$ such that $d_{\lambda}\left(f x_{m}, f x_{m+1}\right)=0$. Applying (3), we get that

$$
\begin{aligned}
\psi_{\lambda}\left(d_{\lambda}\left(f x_{m+1}, f x_{m+2}\right)\right) & \leq \psi_{\lambda}\left(d_{\lambda}\left(g x_{m+1}, g x_{m+2}\right)\right)-\varphi_{\lambda}\left(d_{\lambda}\left(g x_{m+1}, g x_{m+2}\right)\right) \\
& =\psi_{\lambda}\left(d_{\lambda}\left(f x_{m}, f x_{m+1}\right)\right)-\varphi_{\lambda}\left(d_{\lambda}\left(f x_{m}, f x_{m+1}\right)\right) \\
& =\psi_{\lambda}(0)-\varphi_{\lambda}(0)
\end{aligned}
$$

$($ from $(\mathrm{C} 1),(\mathrm{C} 2))=0$.

Then it follows from $(\mathrm{C} 1)$ that $d_{\lambda}\left(f x_{m+1}, f x_{m+2}\right)=0$. Continuing this process, one can show that $d_{\lambda}\left(f x_{n}, f x_{n+1}\right)=0$ for all $n \geq m$. Then our claim (4) holds.

- Second case: We suppose that

$$
d_{\lambda}\left(f x_{n}, f x_{n+1}\right)>0, \quad \text { for all } n \in \mathbb{N} .
$$

Let, if possible, for some $n_{0} \in \mathbb{N}$,

$$
d_{\lambda}\left(f x_{n_{0}-1}, f x_{n_{0}}\right)<d_{\lambda}\left(f x_{n_{0}}, f x_{n_{0}+1}\right) .
$$

By the monotone property of $\psi_{\lambda}$, and using (3), we get

$$
\begin{aligned}
\psi_{\lambda}\left(d_{\lambda}\left(f x_{n_{0}-1}, f x_{n_{0}}\right)\right) & \leq \psi_{\lambda}\left(d_{\lambda}\left(f x_{n_{0}}, f x_{n_{0}+1}\right)\right) \leq \psi_{\lambda}\left(d_{\lambda}\left(g x_{n_{0}}, g x_{n_{0}+1}\right)\right)-\varphi_{\lambda}\left(d_{\lambda}\left(g x_{n_{0}}, g x_{n_{0}+1}\right)\right) \\
& =\psi_{\lambda}\left(d_{\lambda}\left(f x_{n_{0}-1}, f x_{n_{0}}\right)\right)-\varphi_{\lambda}\left(d_{\lambda}\left(f x_{n_{0}-1}, f x_{n_{0}}\right)\right) .
\end{aligned}
$$

Then, by (C2), we have that $d_{\lambda}\left(f x_{n_{0}-1}, f x_{n_{0}}\right)=0$, which contradicts (5). Therefore, we deduce that

$$
d_{\lambda}\left(f x_{n}, f x_{n+1}\right) \leq d_{\lambda}\left(f x_{n-1}, f x_{n}\right), \quad \text { for all } n \geq 1 .
$$

So, it follows that $\left\{d_{\lambda}\left(f x_{n}, f x_{n+1}\right)\right\}$ is a decreasing sequence of non-negative real numbers. Hence, there is $r \geq 0$ such that

$$
d_{\lambda}\left(f x_{n}, f x_{n+1}\right) \rightarrow r \quad \text { as } n \rightarrow+\infty .
$$

On the other hand, from (3), we have

$$
\begin{aligned}
\psi_{\lambda}\left(d_{\lambda}\left(f x_{n}, f x_{n+1}\right)\right) & \leq \psi_{\lambda}\left(d_{\lambda}\left(g x_{n}, g x_{n+1}\right)\right)-\varphi_{\lambda}\left(d_{\lambda}\left(g x_{n}, g x_{n+1}\right)\right) \\
& =\psi_{\lambda}\left(d_{\lambda}\left(f x_{n-1}, f x_{n}\right)\right)-\varphi_{\lambda}\left(d_{\lambda}\left(f x_{n-1}, f x_{n}\right)\right) .
\end{aligned}
$$

This implies that

$$
\limsup _{n \rightarrow \infty} \psi_{\lambda}\left(d_{\lambda}\left(f x_{n}, f x_{n+1}\right)\right) \leq \limsup _{n \rightarrow \infty} \psi_{\lambda}\left(d_{\lambda}\left(f x_{n-1}, f x_{n}\right)\right)-\liminf _{n \rightarrow \infty} \varphi_{\lambda}\left(d_{\lambda}\left(f x_{n-1}, f x_{n}\right)\right) .
$$


Then, using (6), the continuity hypothesis of $\psi_{\lambda}$ and the lower semi-continuity of $\phi_{\lambda}$, we get that

$$
\psi_{\lambda}(r) \leq \psi_{\lambda}(r)-\varphi_{\lambda}(r)
$$

which, by condition (C2) implies that $r=0$. Thus, we proved (4).

Step 2. We will prove that $\left\{x_{n}\right\}$ is a Cauchy sequence in the gauge space $(X, \mathcal{T}(\mathcal{F}))$. Suppose that $\left\{f_{n}\right\}$ is not a Cauchy sequence. Then there exists $(\lambda, \varepsilon) \in \mathcal{A} \times(0, \infty)$ for which we can find two sequences of positive integers $\{m(k)\}$ and $\{n(k)\}$ such that for all positive integers $k$,

$$
n(k)>m(k)>k, \quad d_{\lambda}\left(f x_{m(k)}, f x_{n(k)}\right) \geq \varepsilon, \quad d_{\lambda}\left(f x_{m(k)}, f x_{n(k)-1}\right)<\varepsilon .
$$

Using (7) and the triangular inequality, we get that

$$
\begin{aligned}
\varepsilon & \leq d_{\lambda}\left(f x_{n(k)}, f x_{m(k)}\right) \\
& \leq d_{\lambda}\left(f x_{m(k)}, f x_{n(k)-1}\right)+d_{\lambda}\left(f x_{n(k)-1}, f x_{n(k)}\right) \\
& <\varepsilon+d_{\lambda}\left(f x_{n(k)}, f x_{n(k)-1}\right) .
\end{aligned}
$$

Thus we have

$$
\varepsilon \leq d_{\lambda}\left(f x_{n(k)}, f x_{m(k)}\right)<\varepsilon+d_{\lambda}\left(f x_{n(k)}, f x_{n(k)-1}\right) .
$$

Letting $k \rightarrow+\infty$ in the above inequality and using (4), we obtain

$$
d_{\lambda}\left(f x_{n(k)}, f x_{m(k)}\right) \rightarrow \varepsilon \quad \text { as } k \rightarrow+\infty .
$$

On the other hand, we have

$$
d_{\lambda}\left(f x_{n(k)}, f x_{m(k)}\right) \leq d_{\lambda}\left(f x_{n(k)}, f x_{n(k)-1}\right)+d_{\lambda}\left(f x_{n(k)-1}, f x_{m(k)-1}\right)+d_{\lambda}\left(f x_{m(k)-1}, f x_{m(k)}\right)
$$

and

$$
d_{\lambda}\left(f x_{n(k)-1}, f x_{m(k)-1}\right) \leq d_{\lambda}\left(f x_{n(k)-1}, f x_{n(k)}\right)+d_{\lambda}\left(f x_{n(k)}, f x_{m(k)}\right)+d_{\lambda}\left(f x_{m(k)}, f x_{m(k)-1}\right) .
$$

Thus we have

$$
\left\{\begin{array}{l}
d_{\lambda}\left(f x_{n(k)-1}, f x_{m(k)-1}\right) \geq d_{\lambda}\left(f x_{n(k)}, f x_{m(k)}\right)-d_{\lambda}\left(f x_{n(k)}, f x_{n(k)-1}\right)-d_{\lambda}\left(f x_{m(k)-1}, f x_{m(k)}\right) \\
d_{\lambda}\left(f x_{n(k)-1}, f x_{m(k)-1}\right) \leq d_{\lambda}\left(f x_{n(k)-1}, f x_{n(k)}\right)+d_{\lambda}\left(f x_{n(k)}, f x_{m(k)}\right)+d_{\lambda}\left(f x_{m(k)-1}\right)
\end{array}\right.
$$

which implies that

$$
\left|d_{\lambda}\left(f x_{n(k)-1}, f x_{m(k)-1}\right)-d_{\lambda}\left(f x_{n(k)}, f x_{m(k)}\right)\right| \leq d_{\lambda}\left(f x_{n(k)-1}, f x_{n(k)}\right)+d_{\lambda}\left(f x_{m(k)}, f x_{m(k)-1}\right) .
$$

Letting $k \rightarrow \infty$ in the above inequality, using (4) and (8), we get that

$$
d_{\lambda}\left(f x_{n(k)-1}, f x_{m(k)-1}\right) \rightarrow \varepsilon \quad \text { as } k \rightarrow+\infty .
$$

Applying inequality (3) with $p=n(k)$ and $q=m(k)$, we get that

$$
\psi_{\lambda}\left(d_{\lambda}\left(f x_{n(k)}, f x_{m(k)}\right)\right) \leq \psi_{\lambda}\left(d_{\lambda}\left(g x_{n(k)}, g x_{m(k)}\right)\right)-\varphi_{\lambda}\left(d_{\lambda}\left(g x_{n(k)}, g x_{m(k)}\right)\right),
$$

that is,

$$
\psi_{\lambda}\left(d_{\lambda}\left(f x_{n(k)}, f x_{m(k)}\right)\right) \leq \psi_{\lambda}\left(d_{\lambda}\left(f x_{n(k)-1}, f x_{m(k)-1}\right)\right)-\varphi_{\lambda}\left(d_{\lambda}\left(f x_{n(k)-1}, f x_{m(k)-1}\right)\right) .
$$


Letting $k \rightarrow+\infty$ in the above inequality, using (8), (9), the continuity hypothesis of $\psi_{\lambda}$ and the lower semi-continuity of $\phi_{\lambda}$, we obtain

$$
\psi_{\lambda}(\varepsilon) \leq \psi_{\lambda}(\varepsilon)-\varphi_{\lambda}(\varepsilon),
$$

which implies from $(\mathrm{C} 2)$ that $\varepsilon=0$, which is a contradiction with $\varepsilon>0$. Finally, we deduce that $\left\{f x_{n}\right\}$ is a Cauchy sequence.

Step 3. Existence of a coincidence point.

Since $\left\{f x_{n}\right\}$ is a Cauchy sequence in the complete gauge space $(X, \mathcal{T}(\mathcal{F}))$, then there exists a $z \in X$ such that $f x_{n} \stackrel{\mathcal{F}}{\rightarrow} z$. Since $f$ and $g$ are continuous, we get that $f f x_{n} \stackrel{\mathcal{F}}{\rightarrow} f z$ and $g f x_{n} \stackrel{\mathcal{F}}{\rightarrow} g z$. On the other hand, from $g x_{n+1}=f x_{n}$, we have also $g x_{n} \stackrel{\mathcal{F}}{\rightarrow} z$. Thus, we

$$
f x_{n} \stackrel{\mathcal{F}}{\rightarrow} z, \quad f f x_{n} \stackrel{\mathcal{F}}{\rightarrow} f z, \quad g f x_{n} \stackrel{\mathcal{F}}{\rightarrow} g z, \quad g x_{n} \stackrel{\mathcal{F}}{\rightarrow} z .
$$

From the compatibility hypothesis of the pair $\{f, g\}$, we get that for all $\lambda \in \mathcal{A}$,

$$
d_{\lambda}\left(f g x_{n}, g f x_{n}\right) \rightarrow 0 \quad \text { as } n \rightarrow \infty .
$$

Now, using the triangular inequality, for all $\lambda \in \mathcal{A}$, we have

$$
d_{\lambda}(f z, g z) \leq d_{\lambda}\left(f z, f f x_{n}\right)+d_{\lambda}\left(f g x_{n+1}, g f x_{n+1}\right)+d_{\lambda}\left(g f x_{n+1}, g z\right) .
$$

Letting $n \rightarrow \infty$ in the above inequality, and using (10) and (11), we get that $d_{\lambda}(f z, g z)$ $=0$ for all $\lambda \in \mathcal{A}$. In the virtue of the separating structure of $\mathcal{F}$, this implies that $f z=$ $g z$, that

is, $z$ is a coincidence point of $f$ and $g$.

Let $(X, \mathcal{F}, \preccurlyeq)$ be an ordered gauge space. We consider the following assumption: (H): If $\left\{u_{n}\right\} \subset X$ is a nondecreasing sequence with $u_{n} \stackrel{\mathcal{F}}{\rightarrow} u \in X$, then $u_{n} \preccurlyeq u$ for all $n$.

Theorem 3.2 Let $(X, \mathcal{F}, \preccurlyeq)$ be an ordered complete gauge space satisfying the assumption (H). Let $f, g: X \rightarrow X$ be two mappings such that $f$ is $g$-nondecreasing, $f(X) \subseteq$ $g(X)$ and $g(X)$ is closed. Suppose that

$$
\psi_{\lambda}\left(d_{\lambda}(f x, f y)\right) \leq \psi_{\lambda}\left(d_{\lambda}(g x, g y)\right)-\varphi_{\lambda}\left(d_{\lambda}(g x, g y)\right)
$$

for all $\lambda \in \mathcal{A}$, for all $x, y \in X$ for which $g x \leqslant$ gy. If there exists $x_{0}$ such that $g x_{0} \leqslant f x_{0}$, then $f$ and $g$ have a coincidence point.

Proof. Following the proof of Theorem 3.1, we know that $\left\{g x_{n}\right\}$ is a Cauchy sequence in the ordered complete gauge space $(X, \mathcal{F}, \preccurlyeq)$. Since $g(X)$ is closed, there exists $z \in X$ such that $g x_{n} \stackrel{\mathcal{F}}{\rightarrow} g z$. Then we have

$$
f x_{n} \stackrel{\mathcal{F}}{\rightarrow} g z \quad \text { and } \quad g x_{n} \stackrel{\mathcal{F}}{\rightarrow} g z .
$$

Since $\left\{g x_{n}\right\}$ is a nondecreasing sequence, from $(\mathrm{H})$, we have $g x_{n} \leqslant g z$ for all $n \geq 1$. Then we can apply (12) with $x=x_{n}$ and $y=z$, we obtain

$$
\psi_{\lambda}\left(d_{\lambda}\left(f x_{n}, f z\right)\right) \leq \psi_{\lambda}\left(d_{\lambda}\left(g x_{n}, g z\right)\right)-\varphi_{\lambda}\left(d_{\lambda}\left(g x_{n}, g z\right)\right)
$$

for all $\lambda \in \mathcal{A}$ and $n \geq 1$. Let $\lambda \in \mathcal{A}$ be fixed. Letting $n \rightarrow \infty$ in the above inequality, using (C1), (C2) and (13), we obtain that $\psi_{\lambda}\left(d_{\lambda}(g z, f z)\right)=0$, which implies from (C1) that $d_{\lambda}(g z, f z)=0$. Thus, we proved that $d_{\lambda}(g z, f z)=0$ for all $\lambda \in \mathcal{A}$. Then $g z=f z$ and $z$ is a coincidence point of $g$ and $f$. 
Theorem 3.3 Let $(X, \mathcal{F}, \preccurlyeq)$ be an ordered complete gauge space and $f: X \rightarrow X$ be a nondecreasing mapping. Suppose that

$$
\psi_{\lambda}\left(d_{\lambda}(f x, f y)\right) \leq \psi_{\lambda}\left(d_{\lambda}(x, y)\right)-\varphi_{\lambda}\left(d_{\lambda}(x, y)\right)
$$

for all $(X, \mathcal{F}, \preccurlyeq)$, for all $x, y \in X$ with $x \preccurlyeq y$. Also suppose either

(I) $f$ is continuous or

(II) If $\left\{x_{n}\right\} \subset X$ is a nondecreasing sequence with $x_{n} \stackrel{\mathcal{F}}{\rightarrow} z \in X$, then $x_{n} \leqslant z$ for all $n$.

If there exists $x_{0}$ such that $x_{0} \leqslant f x_{0}$, then $f$ has a fixed point, that is, there exists $z \in X$ such that $z=f z$. Moreover, if $(X, \preccurlyeq)$ is directed, we obtain the uniqueness of the fixed point of $f$.

Proof. The existence of a fixed point of $f$ follows immediately from Theorems 3.1 and 3.2 by taking $g=I_{X}$ (the identity mapping on $X$ ). Now, suppose that $z^{\prime} \in X$ is another fixed point of $f$, that is, $z^{\prime}=f z^{\prime}$. Since $(X, \preccurlyeq)$ is a directed set, there exists $w \in$ $X$ such that $z \leqslant w$ and $z^{\prime} \leqslant w$. Monotonicity of $f$ implies that $f^{n}(z) \leqslant f^{n}(w)$ and $f^{n}\left(z^{\prime}\right) \leqslant f^{n}$ $(w)$. Then we have

$$
\begin{aligned}
\psi_{\lambda}\left(d_{\lambda}\left(z, f^{n}(w)\right)\right) & \leq \psi_{\lambda}\left(d_{\lambda}\left(f^{n-1}(z), f^{n-1}(w)\right)\right)-\varphi_{\lambda}\left(d_{\lambda}\left(f^{n-1}(z), f^{n-1}(w)\right)\right) \\
& \leq \psi_{\lambda}\left(d_{\lambda}\left(f^{n-1}(z), f^{n-1}(w)\right)\right) \\
& =\psi_{\lambda}\left(d_{\lambda}\left(z, f^{n-1}(w)\right)\right) .
\end{aligned}
$$

Since $\psi_{\lambda}$ is a nondecreasing function, we get that

$$
d_{\lambda}\left(z, f^{n}(w)\right) \leq d_{\lambda}\left(z, f^{n-1}(w)\right), \quad \text { for all } n \geq 1, \lambda \in \mathcal{A} .
$$

Then there exists $r_{\lambda} \geq 0$ such that $d_{\lambda}\left(z, f^{\lambda}(w)\right) \rightarrow r_{\lambda}$ as $n \rightarrow \infty$. Letting $n \rightarrow \infty$ in (15), we get that

$$
\psi_{\lambda}\left(r_{\lambda}\right) \leq \psi_{\lambda}\left(r_{\lambda}\right)-\varphi_{\lambda}\left(r_{\lambda}\right)
$$

which implies that $r_{\lambda}=0$. Then we have $f^{n}(w) \stackrel{\mathcal{F}}{\rightarrow} z$. Similarly, one can show that $f^{n}(w) \stackrel{\mathcal{F}}{\rightarrow} z^{\prime}$. Since $(X, \mathcal{T}(\mathcal{F}))$ is Hausdorff, we obtain that $z=z^{\prime}$.

Let $(X, \mathcal{T}(\mathcal{F}))$ be a gauge space and $f, g: X \rightarrow X$ are two giving mappings. For all $x$, $y \in X$ and $\lambda \in \mathcal{A}$, we denote

$$
M_{\lambda}(g x, g y)=\max \left\{d_{\lambda}(g x, g y), d_{\lambda}(g x, f x), d_{\lambda}(g y, f y), \frac{d_{\lambda}(g y, f y)+d_{\lambda}(g y, f x)}{2}\right\} .
$$

We shall prove the following result.

Theorem 3.4 Let $(X, \mathcal{F}, \preccurlyeq)$ be an ordered complete gauge space and let $f, g: X \rightarrow X$ be two continuous mappings such that $f$ is $g$-nondecreasing, $f(X) \subseteq g(X)$ and the pair $\{f$, $g\}$ is compatible. Suppose that

$$
\psi_{\lambda}\left(d_{\lambda}(f x, f y)\right) \leq \psi_{\lambda}\left(M_{\lambda}(g x, g y)\right)-\varphi_{\lambda}\left(M_{\lambda}(g x, g y)\right)
$$

for all $\lambda \in \mathcal{A}$, for all $x, y \in X$ for which $g x \leqslant$ gy. If there exists $x_{0}$ such that $g x_{0} \leqslant f x_{0}$, then $f$ and $g$ have a coincidence point.

Proof. Similarly to the proof of Theorem3.1, we can construct a sequence $\left\{x_{n}\right\}$ in $X$ such that 


$$
g x_{n+1}=f x_{n}, \quad n=0,1, \ldots
$$

for which

$$
g x_{0} \preccurlyeq g x_{1} \preccurlyeq g x_{2} \preccurlyeq \cdots \preccurlyeq g x_{n} \preccurlyeq \cdots
$$

Then from (16), for all $p, q \in \mathbb{N}$, for all $\lambda \in \mathcal{A}$, we have

$$
\psi_{\lambda}\left(d_{\lambda}\left(f x_{p}, f x_{q}\right)\right) \leq \psi_{\lambda}\left(M_{\lambda}\left(g x_{p}, g x_{q}\right)\right)-\varphi_{\lambda}\left(M_{\lambda}\left(g x_{p}, g x_{q}\right)\right) .
$$

We complete the proof in the following three steps.

Step 1. We will prove that

$$
d_{\lambda}\left(f x_{n}, f x_{n+1}\right) \rightarrow 0 \quad \text { as } n \rightarrow+\infty, \text { for all } \lambda \in \mathcal{A} .
$$

Let $\lambda \in \mathcal{A}$. We distinguish two cases.

- First case: We suppose that there exists $m \in \mathbb{N}$ such that $d_{\lambda}\left(f x_{m}, f x_{m+1}\right)=0$. Applying (17), we get that

$$
\psi_{\lambda}\left(d_{\lambda}\left(f x_{m+1}, f x_{m+2}\right)\right) \leq \psi_{\lambda}\left(M_{\lambda}\left(g x_{m+1}, g x_{m+2}\right)\right)-\varphi_{\lambda}\left(M_{\lambda}\left(g x_{m+1}, g x_{m+2}\right)\right) .
$$

A simple computation gives us that

$$
\left.M_{\lambda}\left(g x_{m+1}, g x_{m+2}\right)\right)=d_{\lambda}\left(f x_{m+1}, f x_{m+2}\right) .
$$

Thus, we get that

$$
\psi_{\lambda}\left(d_{\lambda}\left(f x_{m+1}, f x_{m+2}\right)\right) \leq \psi_{\lambda}\left(d_{\lambda}\left(f x_{m+1}, f x_{m+2}\right)\right)-\varphi_{\lambda}\left(d_{\lambda}\left(f x_{m+1}, f x_{m+2}\right)\right),
$$

which implies from (C2) that $d_{\lambda}\left(f x_{m+1}, f x_{m+2}\right)=0$. Continuing this process, one can show that $d_{\lambda}\left(f x_{n}, f x_{n+1}\right)=0$ for all $n \geq m$. Then our claim (18) holds.

- Second case: We suppose that

$$
d_{\lambda}\left(f x_{n}, f x_{n+1}\right)>0, \quad \text { for all } n \in N .
$$

Applying (17), for all $n \geq 1$, we have

$$
\psi_{\lambda}\left(d_{\lambda}\left(f x_{n}, f x_{n+1}\right)\right) \leq \psi_{\lambda}\left(M_{\lambda}\left(g x_{n}, g x_{n+1}\right)\right)-\varphi_{\lambda}\left(M_{\lambda}\left(g x_{n}, g x_{n+1}\right)\right) .
$$

A simple computation gives us that

$$
M_{\lambda}\left(g x_{n}, g x_{n+1}\right)=\max \left\{d_{\lambda}\left(f x_{n-1}, f x_{n}\right), d_{\lambda}\left(f x_{n}, f x_{n+1}\right)\right\} .
$$

If $M_{\lambda}\left(g x_{n}, g x_{n+1}\right)=d_{\lambda}\left(f x_{n}, f x_{n+1}\right)$, we get that

$$
\psi_{\lambda}\left(d_{\lambda}\left(f x_{n}, f x_{n+1}\right)\right) \leq \psi_{\lambda}\left(d_{\lambda}\left(f x_{n}, f x_{n+1}\right)\right)-\varphi_{\lambda}\left(d_{\lambda}\left(f x_{n}, f x_{n+1}\right)\right),
$$

which implies from (C2) that $d_{\lambda}\left(f x_{n}, f x_{n+1}\right)=0$, that is a contradiction with (19). We deduce that $M_{\lambda}\left(g x_{n}, g x_{n+1}\right)=d_{\lambda}\left(f x_{n-1}, f x_{n}\right)$, that is, $d_{\lambda}\left(f x_{n}, f x_{n+1}\right) \leq d_{\lambda}\left(f x_{n-1}, f x_{n}\right)$. So, it follows that $\left\{d_{\lambda}\left(f x_{n-1}, f x_{n}\right)\right\}$ is a decreasing sequence of non-negative real numbers. Hence, there is $r \geq 0$ such that

$$
d_{\lambda}\left(f x_{n-1}, f x_{n}\right) \rightarrow r \quad \text { as } n \rightarrow+\infty .
$$

On the other hand, from (20), we have

$$
\psi_{\lambda}\left(d_{\lambda}\left(f x_{n}, f x_{n+1}\right)\right) \leq \psi_{\lambda}\left(d_{\lambda}\left(f x_{n-1}, f x_{n}\right)\right)-\varphi_{\lambda}\left(d_{\lambda}\left(f x_{n-1}, f x_{n}\right)\right) .
$$


Letting $n \rightarrow \infty$ in the above inequality and using the properties $(\mathrm{C} 1)$ and $(\mathrm{C} 2)$, we get that

$$
\psi_{\lambda}(r) \leq \psi_{\lambda}(r)-\varphi_{\lambda}(r)
$$

which implies from (C2) that $r=0$. Then our claim (18) holds.

Step 2. We will prove that $\left\{x_{n}\right\}$ is a Cauchy sequence in the gauge space $(X, \mathcal{T}(\mathcal{F}))$. Suppose that $\left\{x_{n}\right\}$ is not a Cauchy sequence. Then there exists $(\lambda, \varepsilon) \in \mathcal{A} \times(0, \infty)$ for which we can find two sequences of positive integers $\{m(k)\}$ and $\{n(k)\}$ such that for all positive integers $k$,

$$
n(k)>m(k)>k, \quad d_{\lambda}\left(f x_{m(k)}, f x_{n(k)}\right) \geq \varepsilon, \quad d_{\lambda}\left(f x_{m(k)}, f x_{n(k)-1}\right)<\varepsilon .
$$

As in the proof of Theorem 3.1, one can show that

$$
\lim _{k \rightarrow \infty} d_{\lambda}\left(f x_{n(k)}, f x_{m(k)}\right)=\lim _{k \rightarrow \infty} d_{\lambda}\left(f x_{n(k)-1}, f x_{m(k)-1}\right)=\varepsilon .
$$

Applying inequality (17) with $p=n(k)$ and $q=m(k)$, we get that

$$
\psi_{\lambda}\left(d_{\lambda}\left(f x_{n(k)}, f x_{m(k)}\right)\right) \leq \psi_{\lambda}\left(M_{\lambda}\left(g x_{n(k)}, g x_{m(k)}\right)\right)-\varphi_{\lambda}\left(M_{\lambda}\left(g x_{n(k)}, g x_{m(k)}\right)\right) .
$$

On the other hand, we have

$$
\begin{aligned}
M_{\lambda}\left(g x_{n(k)}, g x_{m(k)}\right)= & \max \left\{d_{\lambda}\left(g x_{n(k)}, g x_{m(k)}\right), d_{\lambda}\left(g x_{n(k)}, f x_{n(k)}\right), d_{\lambda}\left(g x_{m(k)}, f x_{m(k)}\right),\right. \\
& \left.\frac{d_{\lambda}\left(g x_{n(k)}, f x_{m(k)}\right)+d_{\lambda}\left(g x_{m(k)}, f x_{n(k)}\right)}{2}\right\} \\
= & \max \left\{d_{\lambda}\left(f x_{n(k)-1}, f x_{m(k)-1}\right), d_{\lambda}\left(f x_{n(k)-1}, f x_{n(k)}\right), d_{\lambda}\left(f x_{m(k)-1}, f x_{m(k)}\right),\right. \\
& \left.\frac{d_{\lambda}\left(f x_{n(k)-1}, f x_{m(k)}\right)+d_{\lambda}\left(f x_{m(k)-1}, f x_{n(k)}\right)}{2}\right\} .
\end{aligned}
$$

Using the triangular inequality, we get that

$$
\left|d_{\lambda}\left(f x_{n(k)-1}, f x_{m(k)}\right)-d_{\lambda}\left(f x_{n(k)}, f x_{m(k)}\right)\right| \leq d\left(f x_{n(k)-1}, f x_{n(k)}\right)
$$

and

$$
\left|d_{\lambda}\left(f x_{m(k)-1}, f x_{n(k)}\right)-d_{\lambda}\left(f x_{n(k)-1}, f x_{m(k)-1}\right)\right| \leq d\left(f x_{n(k)-1}, f x_{n(k)}\right) .
$$

Letting $k \rightarrow \infty$ in the above inequalities and using (18), (22), we get that

$$
\lim _{k \rightarrow \infty} d_{\lambda}\left(f x_{n(k)-1}, f x_{m(k)}\right)=\lim _{k \rightarrow \infty} d_{\lambda}\left(f x_{m(k)-1}, f x_{n(k)}\right)=\varepsilon .
$$

Now, combining (18), (22), and (24), we obtain

$$
M_{\lambda}\left(g x_{n(k)}, g x_{m(k)}\right) \rightarrow \varepsilon \quad \text { as } k \rightarrow \infty .
$$

Letting $k \rightarrow \infty$ in (23), using (22), (25) and the properties of functions $\psi_{\lambda}$ and $\phi_{\lambda}$, we get that

$$
\psi_{\lambda}(\varepsilon) \leq \psi_{\lambda}(\varepsilon)-\varphi_{\lambda}(\varepsilon),
$$

which implies that $\varepsilon=0$, a contradiction. Finally, we deduce that $\left\{x_{n}\right\}$ is a Cauchy sequence.

Step 3. Existence of a coincidence point. 
Since $\left\{f x_{n}\right\}$ is a Cauchy sequence in the complete gauge space $(X, \mathcal{T}(\mathcal{F}))$, then there exists a $z \in X$ such that $f x_{n} \stackrel{\mathcal{F}}{\rightarrow} z$. The rest part of the proof is similar to that of Theorem 3.1.

Theorem 3.5 Let $(X, \mathcal{F}, \preccurlyeq)$ be an ordered complete gauge space satisfying the assumption (H). Let $f, g: X \rightarrow X$ be two mappings such that $f$ is g-nondecreasing, $f(X) \subseteq$ $g(X)$ and $g(X)$ is closed. Suppose that

$$
\psi_{\lambda}\left(d_{\lambda}(f z, f y)\right) \leq \psi_{\lambda}\left(M_{\lambda}(g x, g y)\right)-\varphi_{\lambda}\left(M_{\lambda}(g x, g y)\right)
$$

for all $\lambda \in \mathcal{A}$, for all $x, y \in X$ for which $g x \leqslant$ gy. If there exists $x_{0}$ such that $g x_{0} \leqslant f x_{0}$, then $f$ and $g$ have a coincidence point.

Proof. It is similar to the proof of Theorem 3.2.

Using the same technique of the proof of Theorem 3.3, we deduce from Theorems 3.4 and 3.5 the following fixed point result.

Theorem 3.6 Let $(X, \mathcal{F}, \preccurlyeq)$ be an ordered complete gauge space and $f: X \rightarrow X$ be a nondecreasing mapping. Suppose that

$$
\psi_{\lambda}\left(d_{\lambda}(f x, f y)\right) \leq\left(\psi_{\lambda}-\varphi_{\lambda}\right)\left(\max \left\{d_{\lambda}(x, y), d_{\lambda}(x, f x), d_{\lambda}(y, f y), \frac{d_{\lambda}(x, f y)+d_{\lambda}(y, f x)}{2}\right\}\right)
$$

for all $\lambda \in \mathcal{A}$, for all $x, y \in X$ with $x \leqslant y$. Also suppose either

(I) $f$ is continuous or

(II) If $\left\{x_{n}\right\} \subset X$ is a nondecreasing sequence with $x_{n} \stackrel{\mathcal{F}}{\rightarrow} z \in X$, then $x_{n} \leqslant z$ for all $n$.

If there exists $x_{0}$ such that $x_{0} \leqslant f \mathrm{x}_{0}$, then $f$ has a fixed point. Moreover, if $(X, \preccurlyeq)$ is directed, we obtain the uniqueness of the fixed point of $f$.

\section{Some consequences}

In this section, we present some fixed point theorems of integral-type on ordered gauge spaces, deduced from our previous obtained results.

Let $\Gamma$ be the set of functions $a:[0, \infty) \rightarrow[0, \infty)$ satisfying

(i) $a$ is locally integrable on $[0, \infty)$.

(ii) For all $\varepsilon>0$, we have $\int_{0}^{\varepsilon} a(t) d t>0$.

Theorem 4.1 Let $(X, \mathcal{F}, \preccurlyeq)$ be an ordered complete gauge space and let $f, g: X \rightarrow X$ be two continuous mappings such that $f$ is $g$-nondecreasing, $f(X) \subseteq g(X)$ and the pair $\{f$, $g$ \} is compatible. Suppose that

$$
\int_{0}^{d_{\lambda}(f x, f y)} a_{\lambda}(t) d t \leq \int_{0}^{d_{\lambda}(g x, g y)} a_{\lambda}(t) d t-\int_{0}^{d_{\lambda}(g x, g y)} b_{\lambda}(t) d t
$$

for all $\lambda \in \mathcal{A}$, for all $x, y \in X$ for which $g x \leqslant$ gy, where $a_{\lambda}, b_{\lambda} \in \Gamma$ for all $\lambda \in \mathcal{A}$. If there exists $x_{0}$ such that $g x_{0} \leqslant f x_{0}$, then $f$ and $g$ have a coincidence point.

Proof. It follows from Theorem 3.1, by taking for all $\lambda \in \mathcal{A}$,

$$
\psi_{\lambda}(t)=\int_{0}^{t} a_{\lambda}(s) d s \quad \text { and } \quad \varphi_{\lambda}(t)=\int_{0}^{t} b_{\lambda}(s) d s, \quad t \geq 0 .
$$


It is clear that for all $\lambda \in \mathcal{A}$, the functions $\psi_{\lambda}$ and $\varphi_{\lambda}$ satisfy conditions (C1) and (C2).

Theorem 4.2 Let $(X, \mathcal{F}, \preccurlyeq)$ be an ordered complete gauge space satisfying the assumption (H). Let $f, g: X \rightarrow X$ be two mappings such that $f$ is $g$-nondecreasing, $f(X) \subseteq$ $g(X)$ and $g(X)$ is closed. Suppose that

$$
\int_{0}^{d_{\lambda}(f x, f y)} a_{\lambda}(t) d t \leq \int_{0}^{d_{\lambda}(g x, g y)} a_{\lambda}(t) d t-\int_{0}^{d_{\lambda}(g x, g y)} b_{\lambda}(t) d t
$$

for all $\lambda \in \mathcal{A}$, for all $x, y \in X$ for which $g x \leqslant$ gy, where $a_{\lambda}, b_{\lambda} \in \Gamma$ for all $\lambda \in \mathcal{A}$. If there exists $x_{0}$ such that $g x_{0} \leqslant f x_{0}$, then $f$ and $g$ have a coincidence point.

Proof. It follows from Theorem 3.2.

Theorem 4.3 Let $(X, \mathcal{F}, \preccurlyeq)$ be an ordered complete gauge space and $f: X \rightarrow X$ be a nondecreasing mapping. Suppose that

$$
\int_{0}^{d_{\lambda}(f x, f y)} a_{\lambda}(t) d t \leq \int_{0}^{d_{\lambda}(x, y)} a_{\lambda}(t) d t-\int_{0}^{d_{\lambda}(x, y)} b_{\lambda}(t) d t
$$

for all $\lambda \in \mathcal{A}$, for all $x, y \in X$ with $x \leqslant y$, where $a_{\lambda}, b_{\lambda} \in \Gamma$ for all $\lambda \in \mathcal{A}$. Also suppose either

(I) $f$ is continuous or

(II) If $\left\{x_{n}\right\} \subset X$ is a nondecreasing sequence with $x_{n} \stackrel{\mathcal{F}}{\rightarrow} z \in X$, then $x_{n} \leqslant z$ for all $n$.

If there exists $x_{0}$ such that $x_{0} \leqslant f x_{0}$, then $f$ has a fixed point. Moreover, if $(X, \preccurlyeq)$ is directed, we obtain the uniqueness of the fixed point of $f$.

Proof. It follows from Theorem 3.3.

Theorem 4.4 Let $(X, \mathcal{F}, \preccurlyeq)$ be an ordered complete gauge space and let $f, g: X \rightarrow X$ be two continuous mappings such that $f$ is $g$-nondecreasing, $f(X) \subseteq g(X)$ and the pair $\{f$, $g\}$ is compatible. Suppose that

$$
\int_{0}^{d_{\lambda}(f x, f y)} a_{\lambda}(t) d t \leq \int_{0}^{M_{\lambda}(g x, g y)} a_{\lambda}(t) d t-\int_{0}^{M_{\lambda}(g x, g y)} b_{\lambda}(t) d t
$$

for all $\lambda \in \mathcal{A}$, for all $x, y \in X$ for which $g x \leqslant$ gy, where $a_{\lambda}, b_{\lambda} \in \Gamma$ for all $\lambda \in \mathcal{A}$. If there exists $x_{0}$ such that $g x_{0} \leqslant f x_{0}$, then $f$ and $g$ have a coincidence point.

Proof. It follows from Theorem 3.4.

Theorem 4.5 Let $(X, \mathcal{F}, \preccurlyeq)$ be an ordered complete gauge space satisfying the assumption (H). Let $f, g: X \rightarrow X$ be two mappings such that $f$ is $g$-nondecreasing, $f(X) \subseteq$ $g(X)$ and $g(X)$ is closed. Suppose that

$$
\int_{0}^{d_{\lambda}(f x, f y)} a_{\lambda}(t) d t \leq \int_{0}^{M_{\lambda}(g x, g y)} a_{\lambda}(t) d t-\int_{0}^{M_{\lambda}(g x, g y)} b_{\lambda}(t) d t
$$

for all $\lambda \in \mathcal{A}$, for all $x, y \in X$ for which $g x \leqslant$ gy, where $a_{\lambda}, b_{\lambda} \in \Gamma$ for all $\lambda \in \mathcal{A}$. If there exists $x_{0}$ such that $g x_{0} \leqslant f x_{0}$, then $f$ and $g$ have a coincidence point.

Proof. It follows from Theorem 3.5. 
Theorem 4.6 Let $(X, \mathcal{F}, \preccurlyeq)$ be an ordered complete gauge space and $f: X \rightarrow X$ be a nondecreasing mapping. Suppose that

$$
\int_{0}^{d_{\lambda}(f x, f y)} a_{\lambda}(t) d t \leq \int_{0}^{M_{\lambda}(x, y)} a_{\lambda}(t) d t-\int_{0}^{M_{\lambda}(x, y)} b_{\lambda}(t) d t
$$

for all $\lambda \in \mathcal{A}$, for all $x, y \in X$ with $x \leqslant y$, where $a_{\lambda}, b_{\lambda} \in \Gamma$ for all $\lambda \in \mathcal{A}$. Also suppose either

(I) $f$ is continuous or

(II) If $\left\{x_{n}\right\} \subset X$ is a nondecreasing sequence with $x_{n} \stackrel{\mathcal{F}}{\rightarrow} z \in X$, then $x_{n} \leqslant z$ for all $n$.

If there exists $x_{0}$ such that $x_{0} \leqslant f x_{0}$, then $f$ has a fixed point. Moreover, if $(X, \preccurlyeq)$ is directed, we obtain the uniqueness of the fixed point of $f$.

Proof. It follows from Theorem 3.6.

\section{Applications}

In this section, we present some examples of nonlinear integral equations, where our obtained results can be applied.

Consider the integral equation

$$
x(t)=\int_{0}^{t} k(t, s, x(s)) d s+h(t), \quad t \geq 0,
$$

where $k:[0, \infty) \times[0, \infty) \times \mathbb{R} \rightarrow \mathbb{R}$ and $h:[0, \infty) \rightarrow \mathbb{R}$.

Previously, we consider the space $X=C([0, \infty), \mathbb{R})$ of real continuous functions defined on $[0, \infty)$. For each positive integer $n \geq 1$, we define the map $\|\cdot\|_{n}: X \rightarrow[0, \infty)$ by

$$
\|x\|_{n}=\max _{0 \leq t \leq n}|x(t)|, \text { for all } x \in X
$$

This map is a semi-norm on $X$. Define now,

$$
d_{n}(x, y)=\|x-y\|_{n^{\prime}} \text { for all } n \geq 1, \quad x, y \in X .
$$

Then $\mathcal{F}=\left\{d_{n}\right\}_{n \geq 1}$ is a separating family of pseudo-metrics on $X$. The gauge space $(X, \mathcal{T}(\mathcal{F}))$ with respect to the family $\mathcal{F}$ is complete. Consider on $X$ the partial order $\leqslant$ defined by

$$
x, y \in X, \quad x \preccurlyeq y \Leftrightarrow x(t) \leq y(t) \text { for all } t \geq 0 .
$$

For any increasing sequence $\left\{x_{n}\right\}$ in $X$ converging to some $z \in X$ we have $x_{n}(t) \leq z(t)$ for any $t \geq 0$. Also, for every $x, y \in X$, there exists $c(x, y) \in X$ which is comparable to $x$ and $y$.

We shall prove the following result.

Theorem 5.1 Suppose that

(i) $k:[0, \infty) \times[0, \infty) \times \mathbb{R} \rightarrow \mathbb{R}$ and $h:[0, \infty) \rightarrow \mathbb{R}$ are continuous;

(ii) $k(t, s, \cdot): \mathbb{R} \rightarrow \mathbb{R}$ is increasing for each $t, s \geq 0$;

(iii) for each $t, s \geq 0, u, v \in \mathbb{R}, u \leq v$, we have

$$
|k(t, s, u)-k(t, s, v)| \leq \gamma(t, s) \sqrt{\ln \left[(v-u)^{2}+1\right]},
$$


where $\gamma:[0, \infty) \times[0, \infty) \rightarrow[0, \infty)$ is continuous, the function $t \mapsto \int_{0}^{t} \gamma(t, s) d s$ is bounded on $[0, \infty)$ and

$$
\sup _{t \geq 0} \int_{0}^{t} \gamma(t, s) d s \leq 1
$$

(iv) there exists $x_{0} \in C([0, \infty), \mathbb{R})$ such that

$$
x_{0}(t) \leq \int_{0}^{t} k\left(t, s, x_{0}(s)\right) d s+h(t), \quad \text { for any } t \geq 0 .
$$

Then the integral equation (26) has a unique solution $x^{* *} \in C([0, \infty), \mathbb{R})$.

Proof. Consider the operator $f: X \rightarrow X$ given by

$$
f x(t)=\int_{0}^{t} k(t, s, x(s)) d s+h(t), \quad t \geq 0, x \in X .
$$

It is clear that $f$ is well defined since $k$ and $h$ are continuous functions.

From condition (ii), for every $x, y \in X$ with $x \leqslant y$, we have

$$
k(t, s, x(s)) \leq k(t, s, y(s)), \quad \text { for all } t, s \geq 0,
$$

which implies that

$$
\int_{0}^{t} k(t, s, x(s)) d s+h(t) \leq \int_{0}^{t} k(t, s, y(s)) d s+h(t), \quad \text { for all } t \geq 0,
$$

that is, $f x \preccurlyeq f y$. This proves that $f$ is a nondecreasing operator.

Taking into account (iii), for each $x, y \in X$ with $x \leqslant y$, for all $t \in[0, n], n \geq 1$, we have

$$
\begin{aligned}
|f x(t)-f y(t)| & \leq \int_{0}^{t}|k(t, s, y(s))-k(t, s, x(s))| d s \\
& \leq \int_{0}^{t} \gamma(t, s) \sqrt{\ln \left[(\gamma(s)-x(s))^{2}+1\right]} d s \\
& \leq \sqrt{\ln \left[\left(d_{n}(x, y)\right)^{2}+1\right]} \int_{0}^{t} \gamma(t, s) d s \\
& \leq \sqrt{\ln \left[\left(d_{n}(x, y)\right)^{2}+1\right]} .
\end{aligned}
$$

Then, for all $n \geq 1$, we have

$$
d_{n}(f x, f y) \leq \sqrt{\ln \left[\left(d_{n}(x, y)\right)^{2}+1\right]}, \quad \text { for all } x, y \in X, \quad x \preccurlyeq y .
$$

Hence, for all $n \geq 1$, we have

$$
\psi_{n}\left(d_{n}(f x, f y)\right) \leq \psi_{n}\left(d_{n}(f x, f y)\right)-\varphi_{n}\left(d_{n}(f x, f y)\right), \quad \text { for all } x, y \in X, \quad x \preccurlyeq y,
$$


where $\psi_{n}(t)=t^{2}$ and $\phi_{n}(t)=t^{2}-\ln \left(t^{2}+1\right)$. Obviously, $\psi_{n}, \phi_{n}$ satisfy the conditions (C1) and (C2). Moreover, from (iv), there exists $x_{0} \in X$ such that $\mathrm{x}_{0} \leqslant f \mathrm{x}_{0}$.

Now, applying Theorem 3.3, we obtain that $f$ has a unique fixed point $x^{*} \in X$, that is, $x^{*} \in C([0, \infty), \mathbb{R})$ is the unique solution to (26).

Consider now the integral equation

$$
x(t)=\int_{-t^{2}}^{t^{2}} k(t, s, x(s)) d s+h(t), \quad t \in \mathbb{R},
$$

where $k: \mathbb{R} \times \mathbb{R} \times \mathbb{R} \rightarrow \mathbb{R}$ and $h: \mathbb{R} \rightarrow \mathbb{R}$.

We consider the space $X=C(\mathbb{R}, \mathbb{R})$ of real continuous functions defined on $\mathbb{R}$. For each positive integer $n \geq 1$, we define the map $\|\cdot\|_{n}: X \rightarrow[0, \infty)$ by

$$
\|x\|_{n}=\max _{-n \leq t \leq n}|x(t)|, \text { for all } x \in X .
$$

This map is a semi-norm on $X$. Define now,

$$
d_{n}(x, y)=\|x-y\|_{n^{\prime}} \text { for all } n \geq 1, \quad x, y \in X .
$$

Then $\mathcal{F}=\left\{d_{n}\right\}_{n \geq 1}$ is a separating family of pseudo-metrics on $X$. The gauge space $(X, \mathcal{T}(\mathcal{F}))$ with respect to the family $\mathcal{F}$ is complete. As before, consider on $X$ the partial order $\leqslant$ defined by

$$
x, y \in X, \quad x \preccurlyeq y \Leftrightarrow x(t) \leq y(t) \text { for all } t \in \mathbb{R} .
$$

For any increasing sequence $\left\{x_{n}\right\}$ in $X$ converging to some $z \in X$ we have $x_{n}(t) \leq z(t)$ for any $t \in \mathbb{R}$. Also, for every $x, y \in X$, there exists $c(x, y) \in X$ which is comparable to $x$ and $y$. We shall prove the following result.

Theorem 5.2 Suppose that

(i) $k: \mathbb{R} \times \mathbb{R} \times \mathbb{R} \rightarrow \mathbb{R}$ and $h: \mathbb{R} \rightarrow \mathbb{R}$ are continuous;

(ii) $k(t, s, \cdot): \mathbb{R} \rightarrow \mathbb{R}$ is increasing for each $t, s \in \mathbb{R}$;

(iii) for each $t, s \in \mathbb{R}, u, v \in \mathbb{R}, u \leq v$, we have

$$
|k(t, s, u)-k(t, s, v)| \leq \gamma(t, s) \sqrt{\ln \left[(v-u)^{2}+1\right]},
$$

where $\gamma: \mathbb{R} \times \mathbb{R} \rightarrow[0, \infty)$ is continuous, the function $t \mapsto \int_{-t^{2}}^{t^{2}} \gamma(t, s) d s$ is bounded on $\mathbb{R}$ and

$$
\sup _{t \in \mathbb{R}} \int_{-t^{2}}^{t^{2}} \gamma(t, s) d s \leq 1
$$

(iv) there exists $x_{0} \in C(\mathbb{R}, \mathbb{R})$ such that

$$
x_{0}(t) \leq \int_{0}^{t} k\left(t, s, x_{0}(s)\right) d s+h(t), \quad \text { for any } t \in \mathbb{R} .
$$

Then the integral equation (27) has a unique solution $x^{*} \in C(\mathbb{R}, \mathbb{R})$. 
Proof. Consider the operator $f: X \rightarrow X$ given by

$$
f x(t)=\int_{-t^{2}}^{t^{2}} k(t, s, x(s)) d s+h(t), \quad t \in R, x \in X .
$$

From condition (ii), for every $x, y \in X$ with $x \preccurlyeq y$, we have

$$
k(t, s, x(s)) \leq k(t, s, y(s)), \quad \text { for all } t, s \in R,
$$

which implies that

$$
\int_{-t^{2}}^{t^{2}} k(t, s, x(s)) d s+h(t) \leq \int_{-t^{2}}^{t^{2}} k(t, s, y(s)) d s+h(t), \quad \text { for all } t \in R,
$$

that is, $f x \preccurlyeq f y$. This proves that $f$ is a nondecreasing operator.

Taking into account (iii), for each $x, y \in X$ with $x \leqslant y$, for all $t \in[-n, n], n \geq 1$, we have

$$
\begin{aligned}
|f x(t)-f y(t)| & \leq \int_{-t^{2}}^{t^{2}}|k(t, s, \gamma(s))-k(t, s, x(s))| d s \\
& \leq \int_{-t^{2}}^{t^{2}} \gamma(t, s) \sqrt{\ln \left[(\gamma(s)-x(s))^{2}+1\right]} d s \\
& \leq \sqrt{\ln \left[\left(d_{n}(x, y)\right)^{2}+1\right]} \int_{-t^{2}}^{t^{2}} \gamma(t, s) d s \\
& \leq \sqrt{\ln \left[\left(d_{n}(x, y)\right)^{2}+1\right]} .
\end{aligned}
$$

Then, for all $n \geq 1$, we have

$$
d_{n}(f x, f y) \leq \sqrt{\ln \left[\left(d_{n}(x, y)\right)^{2}+1\right]}, \quad \text { for all } x, y \in X, \quad x \preccurlyeq y .
$$

Hence, for all $n \geq 1$, we have

$$
\psi_{n}\left(d_{n}(f x, f y)\right) \leq \psi_{n}\left(d_{n}(f x, f y)\right)-\varphi_{n}\left(d_{n}(f x, f y)\right), \quad \text { for all } x, y \in X, \quad x \preccurlyeq y,
$$

where $\psi_{n}(t)=t^{2}$ and $\phi_{n}(t)=t^{2}-\ln \left(t^{2}+1\right)$. Moreover, from (iv), there exists $x_{0} \in X$ such that $x_{0} \leqslant f x_{0}$.

Now, applying Theorem 3.3, we obtain that $f$ has a unique fixed point $x^{*} \in X$ that is, $x^{*} \in C(\mathbb{R}, \mathbb{R})$ is the unique solution to (27).

\section{Author details}

${ }^{1}$ FST Campus Universitaire, 2092, El Manar, Tunis, Tunisia ²Ecole Supérieure des Sciences et Techniques de Tunis, 5, Avenue Taha Hussein-Tunis, B.P. 56, Bab Menara 1008, Tunisie

\section{Authors' contributions}

All authors contributed equally and significantly in writing this article. All authors read and approved the final manuscript.

\section{Competing interests}

The authors declare that they have no competing interests. 


\section{References}

1. Chen, JW, Cho, YJ, Kim, JK, Li, J: Multiobjective optimization problems with modified objective functions and cone constraints and applications. J Global Optim. 49, 137-147 (2011). doi:10.1007/s10898-010-9539-3

2. Choudhury, BS, Kundu, A: $(\psi, a, \beta)$-weak contractions in partially ordered metric spaces. Appl Math Lett. 25, 6-10 (2012). doi:10.1016/j.aml.2011.06.028

3. Harjani, J, Sadarangani, K: Generalized contractions in partially ordered metric spaces and applications to ordinary differential equations. Nonlinear Anal. 72, 1188-1197 (2010). doi:10.1016/j.na.2009.08.003

4. Nieto, JJ, Rodríguez-López, R: Contractive mapping theorems in partially ordered sets and applications to ordinary differential equations. Order. 22, 223-239 (2005). doi:10.1007/s11083-005-9018-5

5. Nieto, JJ, Rodríguez-López, R: Existence and uniqueness of fixed point in partially ordered sets and applications to ordinary differential equations. Acta Math Sin (Engl Ser). 23, 2205-2212 (2007). doi:10.1007/s10114-005-0769-0

6. Ran, ACM, Reurings, MCB: A fixed point theorem in partially orederd sets and some applications to matrix equations. Proc Am Math Soc. 132, 1435-1443 (2004). doi:10.1090/S0002-9939-03-07220-4

7. Banach, S: Sur les opérations dans les ensembles abstraits et leur application aux équations intégrales. Fund Math. 3, 133-181 (1922)

8. Agarwal, RP, Meehan, M, O'Regan, D: Fixed Point Theory and Applications. Cambridge University Press, Cambridge (2001)

9. Ćirić, Lj: Non-self mappings satisfying nonlinear contractive condition with applications. Nonlinear Anal. 71, 2927-2935 (2009). doi:10.1016/j.na.2009.01.174

10. Zeidler, E: Nonlinear Functional Analysis and Its Applications I: Fixed Point Theorems. Springer-Verlag, Berlin (1986)

11. Khan, MS, Swaleh, M, Sessa, S: Fixed point theorems by altering distances between the points. Bull Austral Math Soc. 30(1):1-9 (1984). doi:10.1017/S0004972700001659

12. Albera, Yal, Guerre-Delabriere, S: Principles of weakly contractive maps in Hilbert spaces. In: Gohberg, I, Lyu-bich, Y (eds.) New Results in Operator Theory and its Applications. pp. 7-22. Birkhäuser, Basel (1997). vol. 98 of Operator Theory: Advances and Applications

13. Dorić, D: Common fixed point for generalized ( $\psi$, 区)-weak contractions. Appl Math Lett. 22, 1896-1900 (2009). doi:10.1016/j.aml.2009.08.001

14. Eslamian, M, Abkar, A: A fixed point theorems for generalized weakly contractive mappings in complete metric space. Ital J Pure Appl Math. (in press)

15. Harjani, J, Sadarangani, K: Fixed point theorems for weakly contractive mappings in partially ordered sets. Nonlinear Anal. 71, 3403-3410 (2008)

16. Jachymski, J: Equivalent conditions for generalized contractions on (ordered) metric spaces. Nonlinear Anal. 74, 768-774 (2011). doi:10.1016/j.na.2010.09.025

17. Nashine, HK, Samet, B: Fixed point results for mappings satisfying $(\psi, \nabla)$-weak contractive condition in partially ordered metric spaces. Nonlinear Anal. 74, 2201-2209 (2011). doi:10.1016/j.na.2010.11.024

18. Rhoades, BE: Some theorems on weakly contractive maps. Nonlinear Anal. 47, 2683-2693 (2001). doi:10.1016/S0362546X(01)00388-1

19. Samet, B, Vetro, C, Vetro, P: Fixed point theorems for $a-\psi$-contractive type mappings. Nonlinear Anal. 75, 2154-2165 (2012). doi:10.1016/j.na.2011.10.014

20. Dutta, PN, Choudhury, BS: A generalization of contraction principle in metric spaces. Fixed Point Theory Appl 8 (2008). vol. 2008, Article ID 406368

21. Agarwal, RP, El-Gebeily, MA, O'Regan, D: Generalized contractions in partially ordered metric spaces. Appl Anal. 87, 109-116 (2008). doi:10.1080/00036810701556151

22. Altun, I, Simsek, H: Some fixed point theorems on ordered metric spaces and application. Fixed Point Theory Appl 17 (2010). vol. 2010, Article ID 621492

23. Beg, I, Butt, AR: Fixed point for set-valued mappings satisfying an implicit relation in ordered metric spaces. Nonlinear Anal. 71, 3699-3704 (2009). doi:10.1016/j.na.2009.02.027

24. Bhaskar, TG, Lakshmikantham, V: Fixed point theorems in partially ordered metric spaces and applications. Nonlinear Anal. 65, 1379-1393 (2006). doi:10.1016/j.na.2005.10.017

25. Caballero, J, Harjani, J, Sadarangani, K: Contractive-like mapping principles in ordered metric spaces and application to ordinary differential equations. Fixed Point Theory Appl 14 (2010). vol. 2010, Article ID 916064

26. Chifu, C, Petruşel, G: Fixed-point results for generalized contractions on ordered Gauge spaces with applications. Fixed Point Theory Appl 10 (2011). vol. 2011, Article ID 979586

27. Choudhury, BS, Kundu, A: A coupled coincidence point result in partially ordered metric spaces for compatible mappings. Nonlinear Anal. 73, 2524-2531 (2010). doi:10.1016/j.na.2010.06.025

28. Ćirić, Lj, Agarwal, RP, Samet, B: Mixed monotone-generalized contractions in partially ordered probabilistic metric spaces. Fixed Point Theory Appl 56 (2011). vol. 2011

29. Ćirić, Lj, Cakić, N, Rajović, M, Ume, JS: Monotone generalized nonlinear contractions in partially ordered metric spaces. Fixed Point Theory Appl 11 (2008). vol. 2008, Article ID 131294

30. Cirić, Lj, Lakshmikantham, V: Coupled random fixed point theorems for nonlinear contractions in partially ordered metric spaces. Stoch Anal Appl. 27, 1246-1259 (2009). doi:10.1080/07362990903259967

31. Du, W-S: Coupled fixed point theorems for nonlinear contractions satisfied Mizoguchi-Takahashi's condition in Quasiordered metric spaces. Fixed Point Theory Appl 9 (2010). vol. 2010, Article ID 876372

32. Lakshmikantham, V, Ćirić, Lj: Coupled fixed point theorems for nonlinear contractions in partially ordered metric spaces. Nonlinear Anal. 70, 4341-4349 (2009). doi:10.1016/j.na.2008.09.020

33. O'Regan, D, Petrușel, A: Fixed point theorems for generalized contractions in ordered metric spaces. J Math Anal Appl. 341, 1241-1252 (2008). doi:10.1016/j.jmaa.2007.11.026

34. Radenović, S, Kadelburg, Z: Generalized weak contractions in partially ordered metric spaces. Comput Math Appl. 60, 1776-1783 (2010). doi:10.1016/j.camwa.2010.07.008 
35. Radenović, S, Kadelburg, Z, Jandrlić, D, Jandrlić, A: Some results on weakly contractive maps. Bull Iran Math Soc. (2011, in press)

36. Samet, B: Coupled fixed point theorems for a generalized Meir-Keeler contraction in partially ordered metric spaces. Nonlinear Anal. 72, 4508-4517 (2010). doi:10.1016/j.na.2010.02.026

37. Samet, B, Vetro, C: Coupled fixed point, F-invariant set and fixed point of N-order. Ann Funct Anal. 1, 46-56 (2010)

38. Samet, B, Vetro, C: Coupled fixed point theorems for multi-valued nonlinear contraction mappings in partially ordered metric spaces. Nonlinear Anal. 74, 4260-4268 (2011). doi:10.1016/j.na.2011.04.007

39. Dugundji, J: Topology. Allyn and Bacon, Boston (1966)

doi:10.1186/1687-1812-2012-13

Cite this article as: Cherichi and Samet: Fixed point theorems on ordered gauge spaces with applications to nonlinear integral equations. Fixed Point Theory and Applications 2012 2012:13.

Submit your manuscript to a SpringerOpen ${ }^{\circ}$ journal and benefit from:

- Convenient online submission

- Rigorous peer review

- Immediate publication on acceptance

- Open access: articles freely available online

- High visibility within the field

- Retaining the copyright to your article

Submit your next manuscript at $\boldsymbol{s p r i n g e r o p e n . c o m ~}$ 